\title{
Regulation of PD-L1 expression in a high-grade invasive human oral squamous cell carcinoma microenvironment
}

\author{
MARIKO HIRAI $^{1}$, HIROKO KITAHARA ${ }^{1}$, YUTAKA KOBAYASHI $^{1}$, KOROKU KATO ${ }^{1}$, \\ GEORGE BOU-GHARIOS $^{2}$, HIROYUKI NAKAMURA ${ }^{1}$ and SHUICHI KAWASHIRI ${ }^{1}$ \\ ${ }^{1}$ Department of Oral and Maxillofacial Surgery, Division of Cancer Medicine, \\ Kanazawa University Graduate School of Medical Science, Ishikawa 920-8640, Japan; \\ ${ }^{2}$ Institute of Ageing and Chronic Disease, University of Liverpool, Liverpool L7 8TX, UK
}

Received July 13, 2016; Accepted November 28, 2016

DOI: 10.3892/ijo.2016.3785

\begin{abstract}
Blockade of the programmed-death 1 receptor (PD-1)/programmed-death ligand (PD-L1) pathway efficiently reduces tumour growth and improves survival. Durable tumour regression with blockade of the PD-1/PD-L1 checkpoint has been demonstrated in recent clinical studies. Oral squamous cell carcinoma (OSCC) is highly immunosuppressive, and PD-L1 expression has been proposed as a potential mechanism responsible for this phenotype. Despite the fact that anti-PD-1 treatment can produce durable responses, such therapy appears to benefit only a subset of patients. Thus, it is important to understand the mechanisms underlying regulation of PD-L1 expression in the OSCC microenvironment. In this study, we showed that PD-L1 expression in high-grade invasive OSCC cell lines was lower than that in a low-grade invasive OSCC line and found a close correlation between PD-L1 expression and the epithelial-mesenchymal transition (EMT). PD-L1 expression was upregulated in macrophages and dendritic cells (DCs) in high-grade invasive human OSCC tissues or co-cultured with mesenchymal-phenotype OSCC cells in vitro. TLR4-inhibitory peptide successfully suppressed PD-L1 upregulation on macrophages and DCs co-cultured with mesenchymal-phenotype OSCC cells, suggesting that
\end{abstract}

Correspondence to: Dr Hiroyuki Nakamura, Department of Oral and Maxillofacial Surgery, Kanazawa University Graduate School of Medical Science, 13-1 Takara-machi, Kanazawa, Ishikawa 920-8640, Japan

E-mail: hnak@me.com

Abbreviations: CAF, carcinoma-associated fibroblast; EGFR, epidermal growth factor receptor; EMT, epithelial-mesenchymal transition; HGF, human gingival fibroblast; IFN- $\gamma$, interferon- $\gamma$; NSCLC, non-small cell lung cancer; OSCC, oral squamous cell carcinoma; PD-1, programmed-death 1 receptor; PD-L1, programmeddeath ligand; qPCR, quantitative real-time PCR; TLR4, toll-like receptor 4

Key words: oral cancer, PD-L1, epithelial-mesenchymal transition, tumour microenvironment some EMT-induced tumour antigen is critical for PD-L1 induction on tumour-associated macrophages and DCs. Further studies are necessary to explore the impact of EMT on the tumour immune microenvironment and to identify potential biomarkers for selecting patients who might preferentially benefit from PD-1/PD-L1 blockade or immunotherapies more broadly.

\section{Introduction}

The programmed-death 1 receptor (PD-1, CD279)/ programmed-death ligand 1 (PD-L1, B7-H1, CD274) pathway has emerged as a critical inhibitory pathway that regulates $\mathrm{T}$ cell responses and maintains immune suppression (1-4). Oral squamous cell carcinoma (OSCC) is a malignant tumour found most often in the head and neck region $(5,6)$ that is highly immunosuppressive in general, and PD-L1 expression has been proposed as a potential mechanism facilitating this phenotype (7-9). PD-L1 is expressed in many tumours in a constitutive and interferon- $\gamma($ IFN- $\gamma$ )-inducible manner. PD-L1 binds to an inhibitory receptor (PD-1), which is a member of the B7 family of receptors (4), and to the costimulatory molecule CD80 (B7-1) (10). PD-1 is expressed on activated T cells, and ligation of PD-1 by tumour-associated PD-L1 induces apoptosis or downregulation of effector cytotoxic T lymphocytes (CTL), resulting in an escape from T-cell mediated immune surveillance (11). Blockade of the PD-L1/PD-1 pathway efficiently reduces tumour growth and improves survival (7,12-15). Durable tumour regression with blockade of the PD-1/PD-L1 checkpoint has been demonstrated in recent clinical studies $(1,2)$, leading to the recent registration of an anti-PD-1 antibody for advanced melanoma and non-small cell lung cancer. In addition, the clinical efficacy of an anti-PD-1 monoclonal antibody in OSCC has been observed in phase I studies (16). In general, the response to PD-1 blockade has been commonly correlated with PD-L1 expression in treated tumours (1).

Although anti-PD-1 treatment can produce durable responses, it appears to benefit only a subset of patients. Chen et al recently demonstrated a molecular link between the epithelial-mesenchymal transition (EMT) and intratumoural $\mathrm{CD}^{+} \mathrm{T}$ cell suppression through PD-L1 regulation, in both animal models and human cell lines (17). EMT is a key 
process that drives cancer metastasis and drug resistance, and has been associated with poor prognosis in multiple cancers, including OSCC (18-20). For instance, positive staining for the mesenchymal marker vimentin occurs in specimens from non-small cell lung cancer (NSCLC) patients who develop resistance to epidermal growth factor receptor (EGFR) inhibitors, suggesting that EMT has been triggered in such tumours (21-23). Previously, we found that loss of EGFR expression in OSCC was associated with EMT and might have functional implications in the resistance to cetuximab treatment (24). However, the impact of EMT on reprogramming the tumour immune microenvironment is largely unknown.

To predict the efficacy and optimize anti-PD-1 therapy, alone or in combination, it is important to understand the mechanisms controlling PD-L1 expression. In this study, we focused on the regulation of PD-L1 expression in OSCC, and the mechanism of regulation of PD-L1 expression in the tumour microenvironment.

\section{Materials and methods}

Cell culture. Three human OSCC cell lines established from tumour biopsies with different grades of invasive abilities were used, including OSC-20 cells (low-grade invasive cells), OSC-19 cells (low-grade invasive cells), and TSU cells (highgrade invasive cells). The OSC-20 cell line was derived from a 58-year-old female with tongue cancer metastatic to the cervical lymph nodes (25). OSC-19 was derived from a 61-year-old male with tongue cancer metastatic to the cervical lymph nodes (26). The TSU cell line was established from a patient with gingival squamous cell carcinoma who had developed marked leukocytosis (27). In addition, normal human gingival fibroblasts (HGFs; ATCC no. CRL-2014) obtained from the American Type Culture Collection (Manassas, VA, USA) served as a control. Macrophages and dendritic cells (DCs) were generated from human peripheral blood mononuclear cells (PBMCs), as described previously $(28,29)$. PBMCs were obtained by venepuncture into an 8-ml Vacutainer CPT Cell-Preparation Tube (BD Vacutainer Systems, Franklin Lakes, NJ, USA). Briefly, monocyte-derived macrophages were generated by incubating monocytes $\left(1 \times 10^{6} / \mathrm{ml}\right)$ in RPMI-1640 medium containing $10 \%$ fetal bovine serum (FBS), $2 \mathrm{mM}$ glutamine, $25 \mathrm{mM}$ HEPES, $100 \mathrm{U} / \mathrm{ml}$ penicillin, $100 \mu \mathrm{g} / \mathrm{ml}$ streptomycin, and $50 \mathrm{ng} / \mathrm{ml}$ GM-CSF (PeproTech, Rocky Hill, NJ, USA) at $37^{\circ} \mathrm{C}$ in a $\mathrm{CO}_{2}$ incubator for 7 days. Induced macrophages were examined using an anti-macrophage antibody (MAC387; Abcam, Tokyo, Japan). Monocyte-derived DCs were generated by incubating monocytes at $1 \times 10^{6}$ cells/ml in G4 medium (G4 Dendritic Cell Generation kit; Humanzyme, IL, USA) at $37^{\circ} \mathrm{C}$ in a $\mathrm{CO}_{2}$ (5\%) incubator for 7 days. Induced DCs were examined using an anti-DC antibody (CD83; Abcam). Carcinoma-associated fibroblasts (CAFs) were generated by the addition of recombinant TGF- $\beta 1$ to confluent growth-arrested cell monolayers, as previously described $(30,31)$. Recombinant TGF- $\beta 1$ (R\&D Systems Europe Ltd., Abingdon, UK; 50 ng/ml) was prepared in serum-free culture medium. CAFs were examined using anti- $\alpha$ SMA (R\&D Systems Europe Ltd.) antibodies. Culture inserts $(1-\mu \mathrm{m}$ pore size) were purchased from Corning Japan (Tokyo, Japan). MyD88 homodimerization-inhibitor peptide and toll-like receptor 4 (TLR4)-inhibitory peptide were purchased from Imgenex (San Diego, CA, USA) and Novus Biologicals (Littleton, CO, USA), respectively, and used in the cell-blocking study.

Tissue samples. Experiments using human tissues were approved by the ethics committee of the Kanazawa University Graduate School of Medical Science (IRB no. 2014-004, 352-2), and written informed consent was obtained from patients providing tissue specimens. The subjects included 24 patients with primary OSCC who underwent surgical resection at the Department of Oral and Maxillofacial Surgery at Kanazawa University Hospital between 2000 and 2008. The patients with carcinoma were aged from 43 to 89 years $(64.4 \pm 11.2$ years, mean \pm SD). The grade of tumour differentiation was determined according to the criteria proposed by the World Health Organization (32). The mode of tumour invasion was assessed according to the classification by Yamamoto et al (33).

RNA extraction, cDNA synthesis, and quantitative real-time PCR ( $q P C R)$. The mRNA expression levels of PD-L1, PD-L2, E-cadherin, N-cadherin, Vimentin, and Snaill were analysed using a Rotor-Gene Q 2plex System (Qiagen, Hilden, Germany) with FAM/ZEN/IBFQ probes (Integrated DNA Technologies, Inc., Coralville, IA, USA; DNA sequences not opened). Total RNA was extracted using the RNeasy Protect Mini kit (Qiagen), and cDNA was obtained using the PrimeScript first-strand cDNA Synthesis kit (Takara, Tokyo, Japan). All reactions were performed according to the manufacturer's instructions. We amplified 18S rRNA as an internal standard using HEX/ZEN/IBFQ probes (Integrated DNA Technologies, Inc.; DNA sequences not opened). Relative expression levels were calculated using the $\Delta \Delta C$ t method for qPCR (34), which presents the data as fold-differences in expression level relative to a calibrator sample; in this case, the mean expression of 3 experimental measurements of $18 \mathrm{~S}$ rRNA in control cells or vehicle-treated cells.

Western blot analysis. The cultured cells were lysed with Pierce RIPA buffer (Thermo Scientific, Waltham, MA, USA). Lysates mixed with sample buffer were electrophoretically separated and transferred onto membranes. Membranes were blocked with Blocking One (Nacalai Tesque, Kyoto, Japan), followed by incubations with an anti-PD-L1, E-cadherin, $\mathrm{N}$-cadherin, Vimentin, or Snail1 antibody (Abcam) and an antihuman $\beta$-actin antibody (Cell Signaling Technology, Tokyo, Japan). After washing with Tris-buffered saline (TBS) with $0.05 \%$ Tween, membranes were incubated with a horseradish peroxidase-conjugated anti-mouse IgG. After washing with TBS- $0.05 \%$ Tween, membranes were incubated with the ECL Prime Western Blotting Detection reagent (GE Healthcare, Little Chalfont, UK). Signals were detected and analysed using C-DiGit (M\&S TechnoSystems, Tokyo, Japan).

EMT induction. OSC-20 cells were seeded at $70 \%$ confluence and cultured for 48 and $72 \mathrm{~h}$ in Dulbecco's modified Eagle's medium (Sigma-Aldrich Japan, Tokyo, Japan) with 0.5\% FBS (Hyclone, Logan, UT, USA) to induce EMT. Recombinant human TGF- $\beta 1$ (R\&D Systems, Minneapolis, MN, USA) was added to a final concentration of $5 \mathrm{ng} / \mathrm{ml}$. 
Immunohistochemistry. Each specimen was fixed in $10 \%$ buffered formalin and then embedded in paraffin to prepare serial sections. Immunohistochemical staining was performed using antibodies against PD-L1, Snail, Vimentin, CD83, macrophages (MAC387), $\alpha$ SMA, and fibroblast activation protein (FAP) (Abcam). Primary antibodies were detected using EnVision Single reagents (Dako Japan, Tokyo, Japan), Alexa Fluor 488-labeled goat anti-mouse IgG (Cell Signaling Technology), and Alexa Fluor 546-labeled goat anti-rabbit IgG (Thermo Fisher Scientific, Rockford, IL, USA). Staining specificity was confirmed using the Universal Negative Control for IS-Series Rabbit Primary Antibodies reagent (IS600; Dako Japan) as a negative control, instead of the matching primary antibody. After mounting with ProLong Gold Antifade reagent (Thermo Fisher Scientific, Tokyo, Japan), the tissues were subjected to fluorescence microscopy. Three regions were image-captured under a light microscope at x100 magnification, and the images were evaluated based on number of stained tumour cells, following the method described by Kimura et al (24). PD-L1 expression was evaluated based on the extent of immunolabelling in tumour cell membranes and was classified on a four-point scale: 0 (no labelling, or labelling in $<10 \%$ of tumour cells); 1 (weak labelling, homogeneous or patchy, in $>10 \%$ of tumour cells); 2 (moderate labelling, homogeneous or patchy, in $>10 \%$ of tumour cells); 3 (intense labelling, homogeneous or patchy, in $>10 \%$ of tumour cells). These scores were subsequently grouped into 2 categories: low expression ( 0 or 1$)$ and high expression ( 2 or 3$)$.

Statistical analysis. Statistical analyses were conducted using JMP 12.0 software (SAS Institute, Inc., Cary, NC, USA). The qPCR data are presented as the mean \pm standard error of the mean (SEM). Differences between groups were tested for statistical significance using the 2-tailed Mann-Whitney $\mathrm{U}$ test. Differences were considered significant at $\mathrm{P}<0.05$.

\section{Results}

PD-L1 and PD-L2 expression in 3 different grades of invasive OSCC cell lines. PD-L1 and PD-L2 mRNA expression on 3 human OSCC lines with different grades of invasiveness (OSC-19, OSC-20, and TSU) were analysed. The qPCR data are presented as fold-differences relative to the mean values from 3 experiments with HGF cells (Fig. 1A). Among the 3 OSCC cell lines, the PD-L1 mRNA expression level ranged from 5.73 to 20.48-fold higher than in HGF cells (Fig. 1A), while PD-L2 mRNA expression ranged from 0.38 to 1.48 -fold higher than in HGF cells (Fig. 1A). In particular, the expression of PD-L1 mRNA in low-grade invasive cells (OSC-20 cells) was highest among the 3 OSCC cell lines. Upregulation of PD-L1 mRNA in high-grade invasive cells (TSU cells) was not as remarkable compared to that in the other 2 low-grade invasive cells (OSC-20 and OSC-19 cells). PD-L2 expression on the 3 different invasive human OSCC lines was much lower, and differences were negligible compared to that of PD-L1. We further analysed PD-L1 expression at the protein level. Both low-grade invasive cells (OSC-20 and OSC-19) had a PD-L1 protein expression level higher than that of TSU cells. HGF cells exhibited negligible PD-L1 protein expression (Fig. 1B).
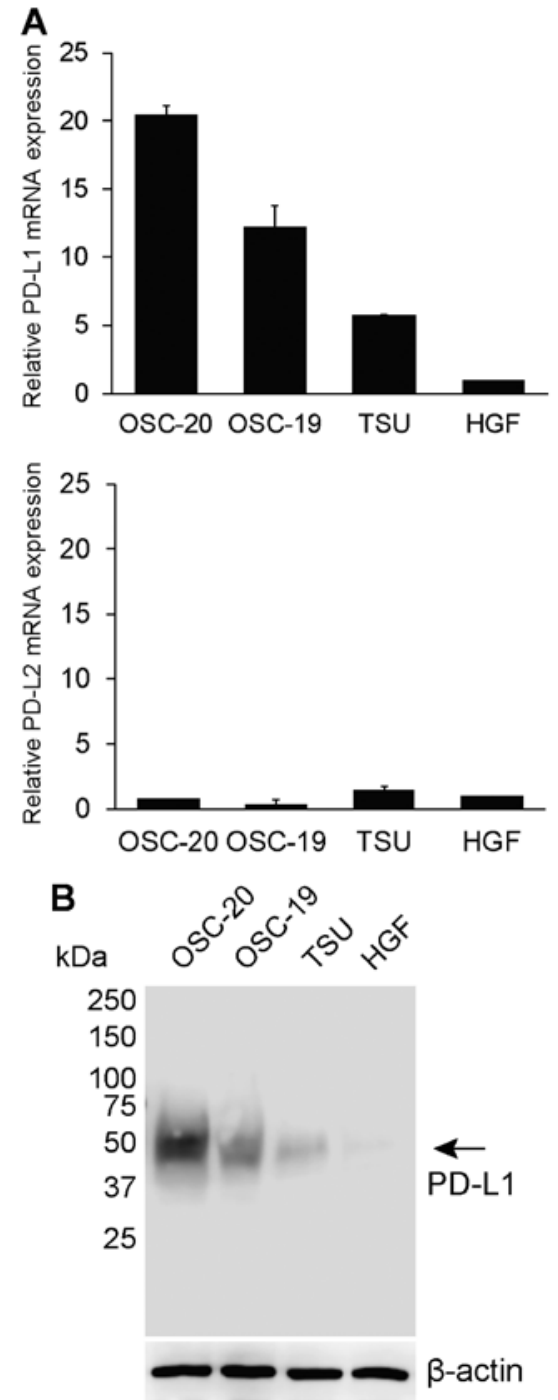

Figure 1. PD-L1 and PD-L2 expression on 3 OSCC cell lines with different grades of invasiveness. (A) Relative mRNA-expression levels of PD-L1 and PD-L2 in the OSC-20, OSC-19, TSU, and HGF cell lines. Expression levels are displayed as fold-differences relative to control cells (HGF cells). (B) Expression of the PD-L1 protein in OSC-20, OSC-19, TSU, and HGF cells was assessed by immunoblot analysis. The arrow indicates PD-L1. $\beta$-actin was detected as a loading control.

The relationship between EMT and PD-L1 expression in human OSCC cell lines. To evaluate whether PD-L1 expression is associated with EMT features, we analysed the mRNA- and protein-expression levels of E-cadherin, $\mathrm{N}$-cadherin, vimentin, and Snail1 in the human OSC-19, OSC-20, and TSU lines with different degrees of invasiveness. The mRNA expression levels of these EMT-associated genes in OSC-19 and TSU cells are presented as fold-differences relative to those in OSC-20 cells. TSU cells displayed a mesenchymal phenotype manifested by the loss of E-cadherin and acquisition of N-cadherin, vimentin, and Snail compared to the corresponding expression levels in OSC-20 and OSC-19 cells (Fig. 2A and B). OSC-20 cells (epithelial phenotype) were exposed to TGF- $\beta 1$ to determine whether such exposure could change the mRNA- and protein-expression levels of EMT-associated genes and PD-L1 (Fig. 2C and D). The mRNA-expression levels are presented as fold-differences relative to that of vehicle control-treated 

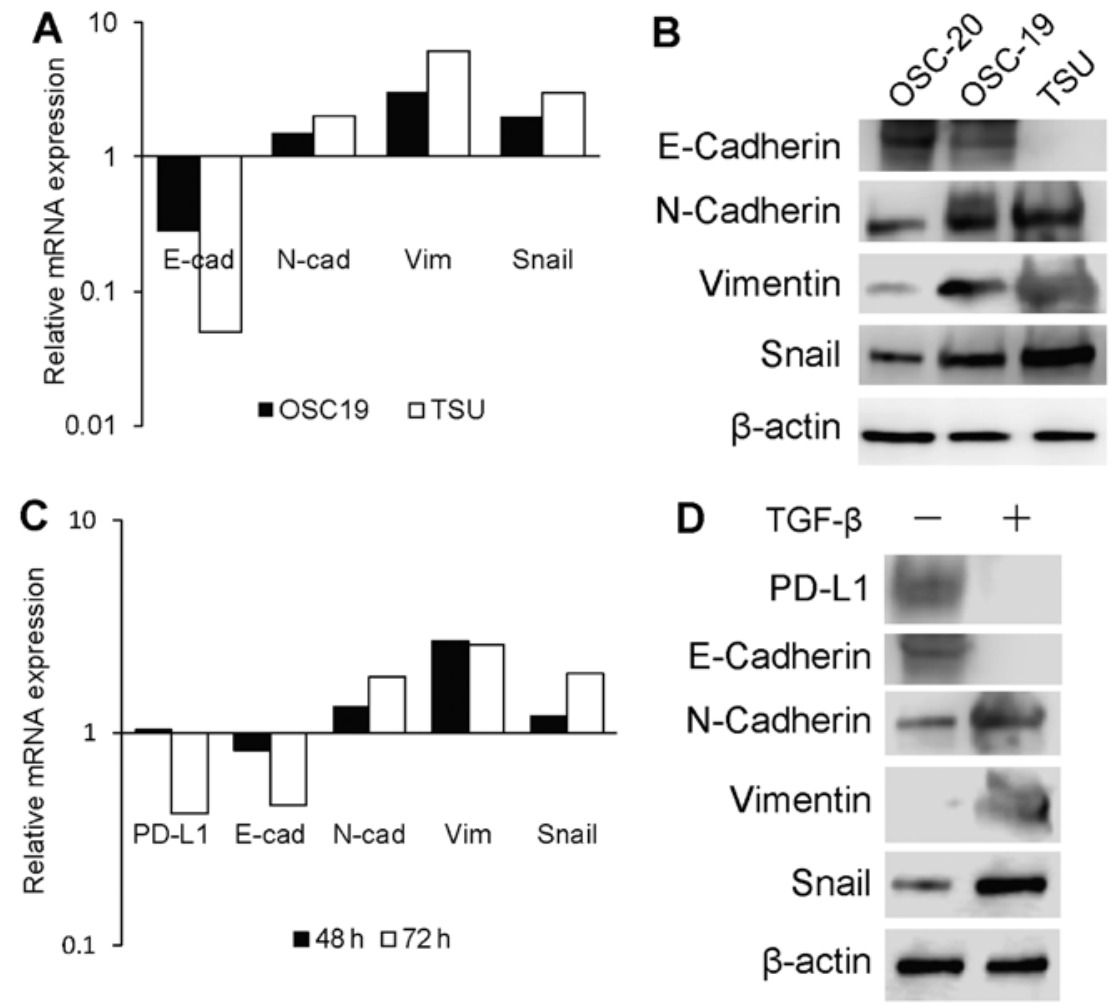

Figure 2. Relationship between EMT and PD-L1 expression on human OSCC cell lines. (A) Relative mRNA expression levels of the EMT-associated genes E-cadherin, N-cadherin, vimentin, and Snail, in OSC-20, OSC-19, and TSU cell lines. Expression differences in OSC-19 and TSU cells are displayed as foldchanges relative to the expression levels in OSC-20 cells. (B) Expression of the EMT-associated protein in OSC-20, OSC-19, and TSU cells was assessed by immunoblot analysis. $\beta$-actin was detected as a loading control. (C) Relative mRNA expression levels of PD-L1 and the EMT-associated genes E-cadherin, $\mathrm{N}$-cadherin, vimentin, and Snail in TGF- $\beta$-treated OSC-20 cells. OSC-20 cells were treated with human TGF- $\beta$ or a vehicle control for 48 and $72 \mathrm{~h}$. Expression differences are displayed as fold-changes relative to the control vehicle at 48 and $72 \mathrm{~h}$. (D) Expression of the EMT-associated protein in TGF- $\beta$-treated OSC-20, OSC-19, and TSU cells was assessed by immunoblot analysis. $\beta$-actin was detected as a loading control.

cells. Serial examination of EMT markers (loss of E-cadherin and upregulation of N-cadherin, vimentin, and Snail) at 48 and $72 \mathrm{~h}$ showed that TGF- $\beta 1$ treatment resulted in EMT in OSC-20 cells (Fig. 2C). We further confirmed E-cadherin downregulation and $\mathrm{N}$-cadherin, vimentin, and Snail upregulation at the protein level at $72 \mathrm{~h}$ after TGF- $\beta 1$ treatment (Fig. 2D). Strikingly, the total PD-L1 mRNA and protein levels were reduced in OSC-20 cells after EMT induction by TGF- $\beta 1$ treatment.

Immunohistochemical analysis of human OSCC tissues. To confirm downregulation of PD-L1 on high-grade invasive cells compared to low-grade invasive cells, we further studied immunohistochemical analysis using 3-, 4C-, and 4D-grade invasive OSCC tissues. Previously, we demonstrated that OSC-20 and OSC-19 cells implanted into nude mice successfully reproduced the same types of diffuse invasive tumours (grades 3 and $4 \mathrm{C}$ in terms of the mode of invasion, respectively) (35), and repeated inoculation with TSU cells successfully reproduced the formation of grade 4D OSCC tumours in nude mice (36). With the low-grade invasive OSCC lines (grades 3 and 4C, mode of invasion), immunohistochemical analysis demonstrated that PD-L1 was detected in cancer cells, but not in stromal cells (Fig. 3A). However, PD-L1 signals were detected in stromal cells of high-grade invasive OSCC (grade 4D, mode of invasion), whereas PD-L1 was not detected in the cancer cells (Fig. 3A). Table I displays the correlations between
PD-L1 expression and the clinicopathological factors. PD-L1 expression in high-grade invasive OSCCs $(4 \mathrm{D}$, mode of invasion) was significantly downregulated compared to low-grade invasive OSCCs (3 and 4C, mode of invasion) $(\mathrm{P}=0.0392)$. In contrast, $\mathrm{PD}-\mathrm{L} 1$ expression did not correlate with tumour size $(\mathrm{P}=0.873)$, lymph node metastasis $(\mathrm{P}=0.542)$, local recurrence $(\mathrm{P}=0.083)$, or histological differentiation $(\mathrm{P}=0.145)$. Immunohistochemical analysis demonstrated that signals of EMT-related protein (Vimentin and Snail1) were detected in cancer cells in human OSCC tissues classified as grade 4D in terms of the mode of invasion (Fig. 3B). To analyse which cells expressed PD-L1 in the cancer stroma, we investigated cells surrounding the cancer cells by studying several biomarkers. Immunofluorescence analysis demonstrated that most CD83 (DC marker)-positive cells expressed PD-L1 and a fraction of MAC387 (macrophage marker)-positive cells expressed the PD-L1 protein in high-grade invasive OSCC tissues (grade 4D, mode of invasion; Fig. 3C). However, $\alpha$ SMA or FAP (CAF marker)-positive cells did not co-localize with PD-L1 expressing cells (Fig. 3C).

PD-L1 expression in macrophages or DCs co-cultured with high-grade, invasive OSCC cells. The above immunohistochemical analysis demonstrated that macrophages and DCs expressed PD-L1 in stromal cells in high-grade invasive OSCC tissues. Thus, we performed further in vitro studies to confirm PD-L1 expression in macrophages and DCs in vitro. We found 
A

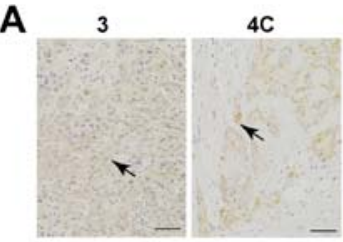

B

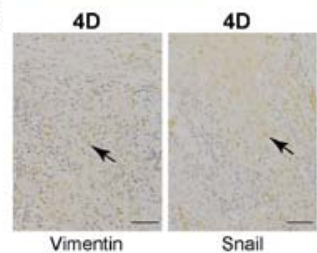

4D

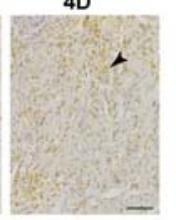

C

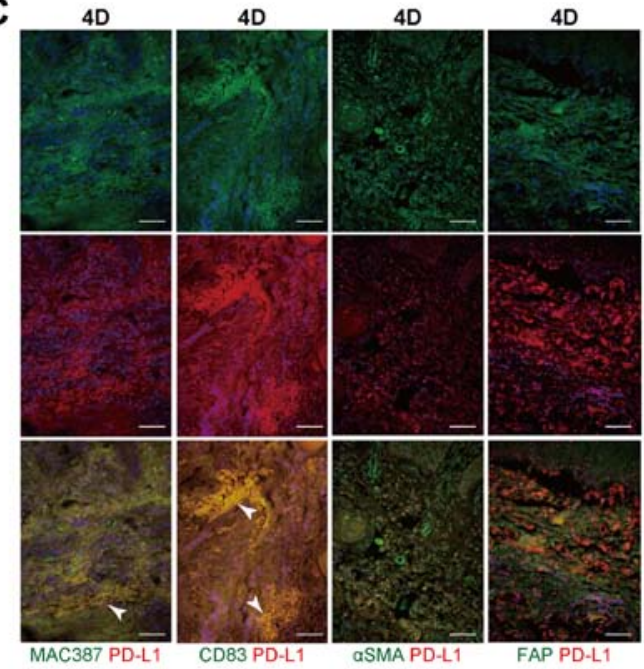

Figure 3. Immunohistochemical analysis of human OSCC tissues. (A) Localization of PD-L1 in OSCC tissues (mode of invasion: grade 3, 4C or 4D). Positive staining of cancer cells (arrow) or stroma cells (arrowhead). (B) Localization of vimentin or snail in OSCC tissues (mode of invasion: grade 4D). Positive staining of cancer cells (arrow). (C) Fluorescent images of MAC387 (green), CD83 (green), $\alpha$ SMA (green), FAP (green), PD-L1 (red), and DAPI (blue) in OSCC tissues (mode of invasion: grade 4D). Double-positive staining of cells (arrowhead). Scale bar, $100 \mu \mathrm{m}$.

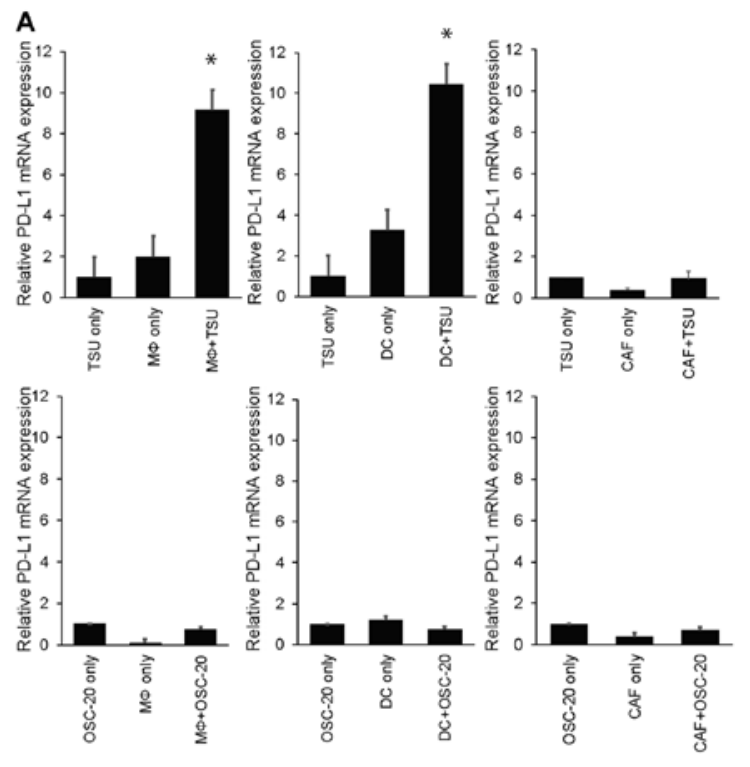

B
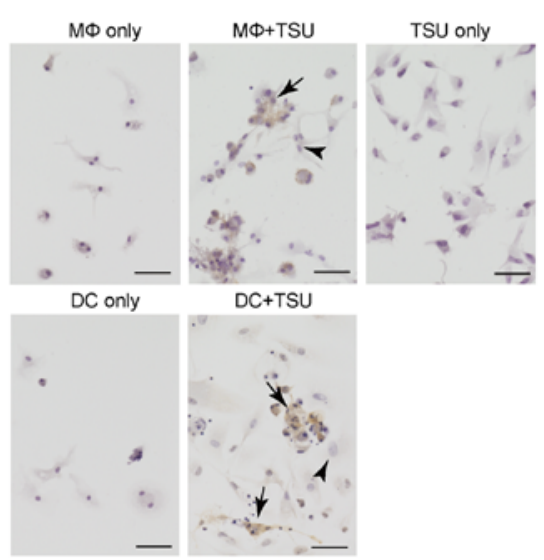

C
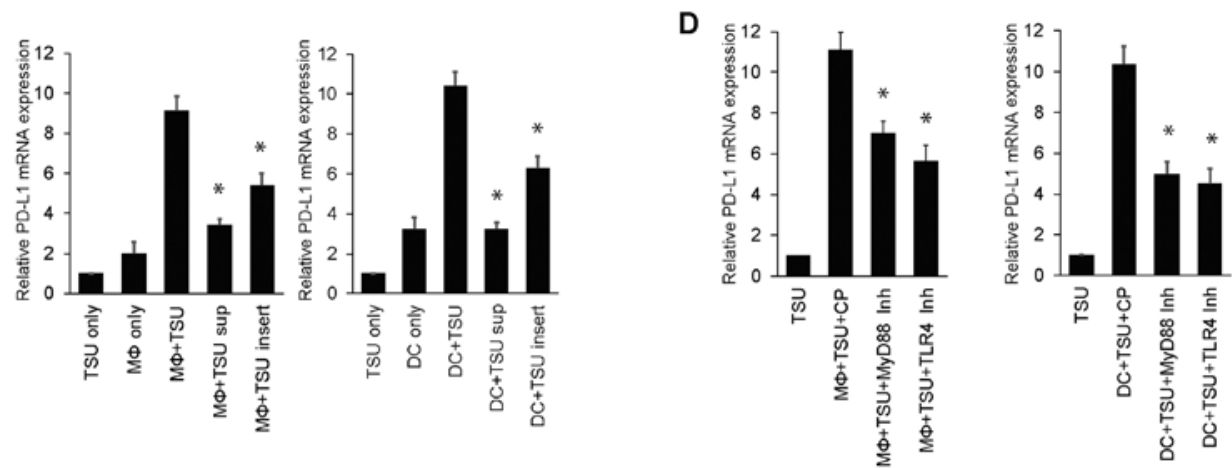

Figure 4. PD-L1 expression on macrophages or DCs co-cultured with high-grade invasive OSCC cells. (A) Expression of PD-L1 mRNA in macrophages or DCs or CAFs co-cultured with TSU or OSC-20 cells. Expression levels are displayed as fold-differences relative to the corresponding mRNA levels in control TSU or OSC-20 cells. The data shown represent the mean \pm SEM. $P<0.05$. (B) Immunohistochemical analysis of macrophages or DCs co-cultured with TSU cells, or macrophages, DCs, and TSU cells cultured separately. Positive immunostaining of macrophages or DCs (arrow) and a lack of staining of TSU cells (arrowhead). Scale bar, $50 \mu \mathrm{m}$. (C) Expression of PD-L1 mRNA in macrophages or DCs after adding the supernatant from a TSU cell culture or TSU cells in a culture insert. Expression levels are displayed as fold-differences relative to the corresponding mRNA levels in control TSU cells. The data shown represent the mean \pm SEM. ${ }^{*} \mathrm{P}<0.05$. (D) PD-L1 upregulation on macrophages or DCs co-cultured with TSU cells was suppressed by a MyD88 homodimerization-inhibitor peptide or TLR4-inhibitory peptide, but not by a control peptide (CP). Expression levels are displayed as fold-differences relative to the corresponding levels observed in control TSU cells. The data shown represent the mean \pm SEM. ${ }^{*} \mathrm{P}<0.05$. 
Table I. Correlation between PD-L1 expression and the OSCC clinicopathological factors of the patients.

\begin{tabular}{|c|c|c|c|}
\hline \multirow[b]{2}{*}{ Factor } & \multicolumn{2}{|c|}{ PD-L1 expression } & \multirow[b]{2}{*}{ P-value } \\
\hline & High $(n=13)$ & Low $(n=11)$ & \\
\hline \multicolumn{4}{|l|}{ T category } \\
\hline $\mathrm{T} 1$ & 4 & 3 & 0.873 \\
\hline $\mathrm{T} 2$ & 7 & 5 & \\
\hline T3 & 0 & 1 & \\
\hline $\mathrm{T} 4$ & 2 & 2 & \\
\hline \multicolumn{4}{|l|}{$\mathrm{N}$ category } \\
\hline Negative & 12 & 7 & 0.542 \\
\hline Positive & 1 & 4 & \\
\hline \multicolumn{4}{|c|}{ Local reccurence } \\
\hline Negative & 10 & 5 & 0.083 \\
\hline Positive & 3 & 6 & \\
\hline \multicolumn{4}{|c|}{ Differentiation } \\
\hline Well & 9 & 7 & 0.145 \\
\hline Moderately & 3 & 1 & \\
\hline Poorly & 1 & 3 & \\
\hline \multicolumn{4}{|c|}{ Mode of invasion } \\
\hline Grade 3 & 6 & 2 & $0.0392^{\mathrm{a}}$ \\
\hline Grade 4C & 5 & 3 & \\
\hline Grade 4D & 2 & 6 & \\
\hline
\end{tabular}

${ }^{\mathrm{a}} \mathrm{P}<0.05$.

that PD-L1 expression increased significantly ( 10 -fold) in macrophages or DCs co-cultured with TSU cells, relative to that observed in TSU cells, macrophages or DCs cultured separately. Differences in PD-L1 expression were negligible between TSU cells co-cultured with CAFs and TSU cells or CAFs cultured separately (Fig. 4A). In addition, PD-L1 expression in macrophages or DCs co-cultured with OSC-20 cells, as well as in separate cultures of OSC-20 cells and macrophages or DCs, did not change (Fig. 4A). Differences in PD-L1 expression were negligible between OSC-20 cells co-cultured with CAFs and OSC-20 cells or CAFs cultured separately (Fig. 4A).

Next, macrophages or DCs co-cultured with TSU cells were immunostained using an anti-PD-L1 antibody to determine which cell type expressed PD-L1. PD-L1 signals were detected in macrophages or DCs, but not TSU cells (Fig. 4B). In particular, macrophages or DCs attached to TSU cells that expressed PD-L1. PD-L1 was not detected in the separate cultures of TSU cells, macrophages, or DCs (Fig. 4B). PD-L1 upregulation was not observed after adding the supernatant from a TSU cell culture to macrophages or DCs (Fig. 4C). PD-L1 upregulation was inhibited after adding TSU cells in culture inserts to macrophages or DCs (Fig. 4C). Further, we used a blocking peptide to study the mechanism of toll-like receptors in PD-L1 induction in macrophages or DCs co-cultured with TSU cells. PD-L1 upregulation in macrophages or DCs co-cultured with TSU cells was significantly suppressed by treatment with the MyD88 homodimerizationinhibitor peptide or TLR4-inhibitory peptide (Fig. 4D).

\section{Discussion}

PD-L1 is a ligand of the inhibitory receptor PD-1, and the PD-L1/PD-1 pathway is involved in the induction and maintenance of peripheral immunotolerance in cancer tissues $(11,37)$. Previous data demonstrated that constitutive expression of PD-L1 occurs in various human cancers, including squamous cell carcinomas of the lung, esophagus, and head and neck; other types of carcinomas of the colon, ovaries, bladder, and breast; melanoma; and glioma (7,12,13,37-45). Tumourassociated PD-L1 is closely correlated with a poor prognosis and/or higher malignancy grade. In general, PD-1 blockade has been commonly correlated with PD-L1 expression in treated tumours (1). Here, we demonstrated that PD-L1 expression in 3 OSCC cell lines was significantly upregulated compared to that in normal cells; thus, PD-L1 may play a greater role in immunotolerance in the cancer microenvironment than PD-L2 does. However, it was surprising that PD-L1 expression in high-grade invasive OSCC cell lines was lower than that in a low-grade invasive OSCC line because these data contradicted previous findings with other tumour types $(7,12,13,37-45)$. While the molecular mechanisms that account for this difference remain to be determined, we suggest that they may be confined to cancer subtypes with a unique molecular profile. Clearly, further investigation will be required to test this hypothesis.

Previously, we demonstrated that high-grade invasive OSCC cells have a mesenchymal phenotype (24). In this study, we confirmed the EMT-associated genes were upregulated in high-grade invasive OSCC tumour cells (TSU). Interestingly, PD-L1 expression in TSU OSCC cells (with a mesenchymal phenotype) was downregulated compared to that in OSC-19 and OSC-20 cells (with an epithelial phenotype). We also demonstrated that TGF- $\beta 1$ caused OSC-20 cells (with an epithelial phenotype) to undergo an EMT-associated gene switch, resulting in lost PD-L1 expression. These results indicated that a close correlation existed between PD-L1 expression and EMT, and that the net effect of TGF- $\beta 1$ signalling was PD-L1 downregulation with a concomitant EMT-associated gene switch in tumour cells. However, further studies are needed to explain why OSCC maintains high-grade invasive ability even after PD-L1 downregulation in cancer cells. Our above data were developed in cell line models, which do not reflect contributions of the tumour microenvironment and immune responses, which play important roles in therapeutic responses. Further studies of the precise molecular mechanisms underlying the association between EMT and the regulation of PD-L1 expression in a human tumour microenvironment should be conducted.

By analysing the immunomarkers PD-L1, vimentin, and snaill, we confirmed the loss of PD-L1 expression and the mesenchymal phenotype of cancer cells in high-grade invasive human OSCC tissues (grade 4D). Interestingly, strong PD-L1 signals were detected in stromal cells of high-grade invasive human OSCC tissues (grade 4D), rather than in cancer cells. In low-grade invasive human OSCC tissues (grade 3 and 4C), PD-L1 expression was seen only in cancer cells, but not in 
stromal cells. These data suggested that invasiveness and the EMT signature were inversely correlated with PD-L1 expression in cancer cells and directly correlated with PD-L1 expression in stromal cells. Potentially, some interaction occurred between EMT-induced OSCC cells and stromal cells. A previous report provided evidence that PD-L1 is not only expressed by tumour cells, but also by tumour-associated macrophages, DCs, and CAFs $(46,47)$. In this study, we detected a strong presence of PD-L1 in macrophages or DCs in cancer stroma tissues via immunofluorescence analysis. While the expression levels of PD-L1 in tumour cells, tumour-infiltrating immune cells have recently been shown to correlate with clinical responses to anti-PD-1 therapy (48-50). Only a subset of patients with PD-L1expressing tumours had clinical responses and other patients (without PD-L1 staining) demonstrated clinical benefits, indicating that additional factors in the tumour microenvironment (such as stroma cell PD-L1 expression) may exist that define the subgroup of patients who derive benefit.

Here, we showed that macrophages or DCs co-localized with PD-L1 protein expression in cancer stromal tissues by immunofluorescence staining. We performed further in vitro analysis of the effects of direct interactions between cancer cells and macrophages or DCs on PD-L1 expression. We showed here that PD-L1 expression was upregulated in macrophages or DCs co-cultured with TSU cells, but upregulation was not observed in CAFs co-cultured with TSU cells. Immunostaining of co-cultured macrophages or DCs and TSU cells revealed that PD-L1 upregulation was observed only in macrophages or DCs, but not in co-cultured TSU cells. Although the association between PD-L1 expression and macrophages or DCs reported here is suggestive of an adaptive resistance mechanism, it is still necessary to explain why PD-L1 was predominantly expressed by macrophages or DCs, rather than cancer cells. If cancer cell-derived IFN- $\gamma$ is indeed a major mediator of PD-L1 expression in oral cancer, as described in other settings (51), then oral cancer cells may be less IFN- $\gamma$-responsive than macrophages or DCs. Another possibility is that IFN- $\gamma$ is expressed at lower levels in TSU cells, as we showed here that the supernatant of cultured TSU cells or TSU cells in culture insert did not induce PD-L1 expression in macrophages or DCs. Alternatively, perhaps while cleaning up tumour debris, macrophages and DCs begin to present high levels of tumour antigens, making them prime targets for $\mathrm{T}$ cell recognition. In fact, in this study, a TLR4-inhibitory peptide successfully suppressed PD-L1 upregulation in macrophages or DCs co-cultured with TSU cells. In this scenario, PD-L1 expression may provide a means for protecting macrophages or DCs against cell death, and tumour antigen could be induced by EMT, because PD-L1 upregulation was not observed in macrophages or DCs co-cultured with OSC-20 cells with an epithelial phenotype (Fig. 4A).

\section{Acknowledgements}

This study was supported by grants-in-aid for Scientific Research from the Ministry of Education, Science, Sports and Culture, Japan (nos. 15K20510 and 20390504 to H.K., no. 26463038 to K.K., no. 25462882 to H.N., no. $15 \mathrm{H} 05042$ to S.K.). We are grateful to the members of the Department of Oral and Maxillofacial Surgery of Kanazawa University for their helpful suggestions and assistance. We thank Elsevier Premium Language Editing Services for assistance with language editing.

\section{References}

1. Topalian SL, Hodi FS, Brahmer JR, Gettinger SN, Smith DC, McDermott DF, Powderly JD, Carvajal RD, Sosman JA, Atkins MB, et al: Safety, activity, and immune correlates of anti-PD-1 antibody in cancer. N Engl J Med 366: 2443-2454, 2012.

2. Brahmer JR, Tykodi SS, Chow LQ, Hwu WJ, Topalian SL, Hwu P, Drake CG, Camacho LH, Kauh J, Odunsi K, et al: Safety and activity of anti-PD-L1 antibody in patients with advanced cancer. N Engl J Med 366: 2455-2465, 2012.

3. Topalian SL, Drake CG and Pardoll DM: Targeting the PD-1/ B7-H1(PD-L1) pathway to activate anti-tumor immunity. Curr Opin Immunol 24: 207-212, 2012.

4. Freeman GJ, Long AJ, Iwai Y, Bourque K, Chernova T, Nishimura H, Fitz LJ, Malenkovich N, Okazaki T, Byrne MC, et al: Engagement of the PD-1 immunoinhibitory receptor by a novel B7 family member leads to negative regulation of lymphocyte activation. J Exp Med 192: 1027-1034, 2000.

5. Oliveira-Neto HH, Gleber-Netto FO, de Sousa SF, França CM, Aguiar MC, Silva TA and Batista AC: A comparative study of microvessel density in squamous cell carcinoma of the oral cavity and lip. Oral Surg Oral Med Oral Pathol Oral Radiol 113: 391-398, 2012.

6. Liang X, Zhou H, Liu X, He Y, Tang Y, Zhu G, Zheng M and Yang J: Effect of local hyperthermia on lymphangiogenic factors VEGF-C and -D in a nude mouse xenograft model of tongue squamous cell carcinoma. Oral Oncol 46: 111-115, 2010.

7. Strome SE, Dong H, Tamura H, Voss SG, Flies DB, Tamada K, Salomao D, Cheville J, Hirano F, Lin W, et al: B7-H1 blockade augments adoptive T-cell immunotherapy for squamous cell carcinoma. Cancer Res 63: 6501-6505, 2003.

8. Lyford-Pike S, Peng S, Young GD, Taube JM, Westra WH, Akpeng B, Bruno TC, Richmon JD, Wang H, Bishop JA, et al: Evidence for a role of the PD-1:PD-L1 pathway in immune resistance of HPV-associated head and neck squamous cell carcinoma. Cancer Res 73: 1733-1741, 2013.

9. Zandberg DP and Strome SE: The role of the PD-L1:PD-1 pathway in squamous cell carcinoma of the head and neck. Oral Oncol 50: 627-632, 2014.

10. Butte MJ, Keir ME, Phamduy TB, Sharpe AH and Freeman GJ: Programmed death-1 ligand 1 interacts specifically with the B7-1 costimulatory molecule to inhibit $\mathrm{T}$ cell responses. Immunity 27 : 111-122, 2007.

11. Keir ME, Butte MJ, Freeman GJ and Sharpe AH: PD-1 and its ligands in tolerance and immunity. Annu Rev Immunol 26: 677-704, 2008.

12. Dong H, Strome SE, Salomao DR, Tamura H, Hirano F, Flies DB, Roche PC, Lu J, Zhu G, Tamada K, et al: Tumor-associated B7-H1 promotes T-cell apoptosis: A potential mechanism of immune evasion. Nat Med 8: 793-800, 2002.

13. Tsushima F, Tanaka K, Otsuki N, Youngnak P, Iwai H, Omura K and Azuma M: Predominant expression of B7-H1 and its immunoregulatory roles in oral squamous cell carcinoma. Oral Oncol 42: 268-274, 2006

14. Iwai Y, Terawaki S and Honjo T: PD-1 blockade inhibits hematogenous spread of poorly immunogenic tumor cells by enhanced recruitment of effector T cells. Int Immunol 17: 133-144, 2005.

15. Hirano F, Kaneko K, Tamura H, Dong H, Wang S, Ichikawa M, Rietz C, Flies DB, Lau JS, Zhu G, et al: Blockade of B7-H1 and PD-1 by monoclonal antibodies potentiates cancer therapeutic immunity. Cancer Res 65: 1089-1096, 2005.

16. Ibrahim R, Stewart R and Shalabi A: PD-L1 blockade for cancer treatment: MEDI4736. Semin Oncol 42: 474-483, 2015.

17. Chen L, Gibbons DL, Goswami S, Cortez MA, Ahn YH, Byers LA, Zhang X, Yi X, Dwyer D, Lin W, et al: Metastasis is regulated via microRNA-200/ZEB1 axis control of tumour cell PD-L1 expression and intratumoral immunosuppression. Nat Commun 5: 5241, 2014.

18. Prudkin L, Liu DD, Ozburn NC, Sun M, Behrens C, Tang X, Brown KC, Bekele BN, Moran C and Wistuba II: Epithelial-tomesenchymal transition in the development and progression of adenocarcinoma and squamous cell carcinoma of the lung. Mod Pathol 22: 668-678, 2009. 
19. Zavadil J, Haley J, Kalluri R, Muthuswamy SK and Thompson E: Epithelial-mesenchymal transition. Cancer Res 68: 9574-9577, 2008.

20. Chaffer CL and Weinberg RA: A perspective on cancer cell metastasis. Science 331: 1559-1564, 2011

21. Uramoto H, Iwata T, Onitsuka T, Shimokawa H, Hanagiri T and Oyama T: Epithelial-mesenchymal transition in EGFR-TKI acquired resistant lung adenocarcinoma. Anticancer Res 30: 2513-2517, 2010

22. Chung JH, Rho JK, Xu X, Lee JS, Yoon HI, Lee CT, Choi YJ, $\mathrm{Kim} \mathrm{HR}, \mathrm{Kim} \mathrm{CH}$ and Lee JC: Clinical and molecular evidences of epithelial to mesenchymal transition in acquired resistance to EGFR-TKIs. Lung Cancer 73: 176-182, 2011

23. Sequist LV, Waltman BA, Dias-Santagata D, Digumarthy $S$, Turke AB, Fidias P, Bergethon K, Shaw AT, Gettinger S, Cosper AK, et al: Genotypic and histological evolution of lung cancers acquiring resistance to EGFR inhibitors. Sci Transl Med 3: 75ra26, 2011.

24. Kimura I, Kitahara H, Ooi K, Kato K, Noguchi N, Yoshizawa K, Nakamura $\mathrm{H}$ and Kawashiri S: Loss of epidermal growth factor receptor expression in oral squamous cell carcinoma is associated with invasiveness and epithelial-mesenchymal transition. Oncol Lett 11: 201-207, 2016

25. Yokoi T, Hirata S, Nishimura F, Miyakawa A, Odajima T and Kohama G and Mochizuki Y: Some properties of a newly established human cell line derived from an oral squamous carcinoma. Tumor Res 25: 93-103, 1990.

26. Yokoi T, Homma $\mathrm{H}$ and Odajima T: Establishment and characterization of OSC-19 cell line in serum and protein free culture. Tumor Res 24: 1-17, 1988

27. Hayashi E, Rikimaru K and Nagayama M: Simultaneous production of G- and M-CSF by an oral cancer cell line and the synergistic effects on associated leucocytosis. Eur J Cancer B Oral Oncol 31B: 323-327, 1995.

28. Arrighi JF, Hauser C, Chapuis B, Zubler RH and Kindler V: Long-term culture of human CD34(+) progenitors with FLT3ligand, thrombopoietin, and stem cell factor induces extensive amplification of a CD34(-)CD14(-) and a CD34(-)CD14(+) dendritic cell precursor. Blood 93: 2244-2252, 1999.

29. Yang D, Chen Q, Le Y, Wang JM and Oppenheim JJ: Differential regulation of formyl peptide receptor-like 1 expression during the differentiation of monocytes to dendritic cells and macrophages. J Immunol 166: 4092-4098, 2001.

30. Evans RA, Tian YC, Steadman R and Phillips AO: TGF-beta1mediated fibroblast-myofibroblast terminal differentiation - the role of Smad proteins. Exp Cell Res 282: 90-100, 2003.

31. Adam L, Zhong M, Choi W, Qi W, Nicoloso M, Arora A, Calin G, Wang H, Siefker-Radtke A, McConkey D, et al: miR-200 expression regulates epithelial-to-mesenchymal transition in bladder cancer cells and reverses resistance to epidermal growth factor receptor therapy. Clin Cancer Res 15: 5060-5072, 2009.

32. Thompson L: World Health Organization classification of tumours: Pathology and genetics of head and neck tumours. Ear Nose Throat J 85: 74, 2006.

33. Yamamoto E, Kohama G, Sunakawa H, Iwai M and Hiratsuka $H$ : Mode of invasion, bleomycin sensitivity, and clinical course in squamous cell carcinoma of the oral cavity. Cancer 51: 2175-2180, 1983.

34. Livak KJ and Schmittgen TD: Analysis of relative gene expression data using real-time quantitative PCR and the 2(-Delta Delta C(T)) method. Methods 25: 402-408, 2001.

35. Kawashiri S, Kumagai S, Kojima K, Harada $\mathrm{H}$ and Yamamoto E: Development of a new invasion and metastasis model of human oral squamous cell carcinomas. Eur J Cancer B Oral Oncol 31B: 216-221, 1995

36. Moriyama M: Development of diffuse invasive (grade 4D) human oral squamous cell carcinoma model in severe combined immunodeficiency mice: Microangioarchitectural analysis and immunohistochemical study. Oral Oncol 35: 395-400, 1999.
37. Zou W and Chen L: Inhibitory B7-family molecules in the tumour microenvironment. Nat Rev Immunol 8: 467-477, 2008.

38. Wintterle S, Schreiner B, Mitsdoerffer M, Schneider D, Chen L, Meyermann R, Weller M and Wiendl H: Expression of the B7-related molecule B7-H1 by glioma cells: A potential mechanism of immune paralysis. Cancer Res 63: 7462-7467, 2003.

39. Konishi J, Yamazaki K, Azuma M, Kinoshita I, Dosaka-Akita H and Nishimura M: B7-H1 expression on non-small cell lung cancer cells and its relationship with tumor-infiltrating lymphocytes and their PD-1 expression. Clin Cancer Res 10: 5094-5100, 2004.

40. Ohigashi Y, Sho M, Yamada Y, Tsurui Y, Hamada K, Ikeda N, Mizuno T, Yoriki R, Kashizuka H, Yane K, et al: Clinical significance of programmed death-1 ligand-1 and programmed death-1 ligand-2 expression in human esophageal cancer. Clin Cancer Res 11: 2947-2953, 2005.

41. Thompson RH and Kwon ED: Significance of B7-H1 overexpression in kidney cancer. Clin Genitourin Cancer 5: 206-211, 2006.

42. Hamanishi J, Mandai M, Iwasaki M, Okazaki T, Tanaka Y, Yamaguchi K, Higuchi T, Yagi H, Takakura K, Minato N, et al: Programmed cell death 1 ligand 1 and tumor-infiltrating CD8 ${ }^{+}$ $\mathrm{T}$ lymphocytes are prognostic factors of human ovarian cancer. Proc Natl Acad Sci USA 104: 3360-3365, 2007.

43. Nomi T, Sho M, Akahori T, Hamada K, Kubo A, Kanehiro H, Nakamura S, Enomoto K, Yagita H, Azuma M, et al: Clinical significance and therapeutic potential of the programmed death-1 ligand/programmed death-1 pathway in human pancreatic cancer. Clin Cancer Res 13: 2151-2157, 2007.

44. Ghebeh H, Tulbah A, Mohammed S, Elkum N, Bin Amer SM, Al-Tweigeri T and Dermime S: Expression of B7-H1 in breast cancer patients is strongly associated with high proliferative Ki-67-expressing tumor cells. Int J Cancer 121: 751-758, 2007.

45. Yao Y, Tao R, Wang X, Wang Y, Mao Y and Zhou LF: B7-H1 is correlated with malignancy-grade gliomas but is not expressed exclusively on tumor stem-like cells. Neuro-oncol 11: 757-766, 2009.

46. Okazaki T, Chikuma S, Iwai Y, Fagarasan S and Honjo T: A rheostat for immune responses: The unique properties of PD-1 and their advantages for clinical application. Nat Immunol 14: 1212-1218, 2013.

47. Nazareth MR, Broderick L, Simpson-Abelson MR, Kelleher RJ Jr, Yokota SJ and Bankert RB: Characterization of human lung tumor-associated fibroblasts and their ability to modulate the activation of tumor-associated T cells. J Immunol 178: 5552-5562, 2007.

48. Taube JM, Klein A, Brahmer JR, Xu H, Pan X, Kim JH, Chen L, Pardoll DM, Topalian SL and Anders RA: Association of PD-1, PD-1 ligands, and other features of the tumor immune microenvironment with response to anti-PD-1 therapy. Clin Cancer Res 20: 5064-5074, 2014

49. Herbst RS, Soria JC, Kowanetz M, Fine GD, Hamid O, Gordon MS Sosman JA, McDermott DF, Powderly JD, Gettinger SN, et al: Predictive correlates of response to the anti-PD-L1 antibody MPDL3280A in cancer patients. Nature 515: 563-567, 2014.

50. Garon EB, Rizvi NA, Hui R, Leighl N, Balmanoukian AS, Eder JP, Patnaik A, Aggarwal C, Gubens M, Horn L, et al; KEYNOTE-001 Investigators: Pembrolizumab for the treatment of non-small-cell lung cancer. N Engl J Med 372: 2018-2028, 2015.

51. Taube JM, Anders RA, Young GD, Xu H, Sharma R, McMiller TL, Chen S, Klein AP, Pardoll DM, Topalian SL, et al: Colocalization of inflammatory response with B7-h1 expression in human melanocytic lesions supports an adaptive resistance mechanism of immune escape. Sci Transl Med 4: 127ra37, 2012. 